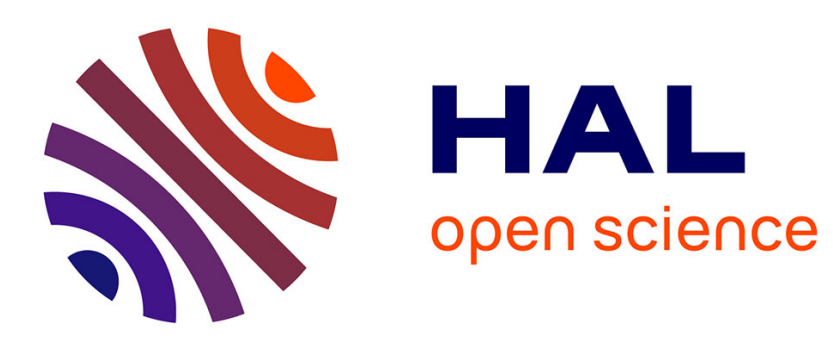

\title{
Templates for engineered nano-objects for use in microwave, electronic devices and biomedical sensing application
}

E. Ferain, R. Legras

\section{- To cite this version:}

E. Ferain, R. Legras. Templates for engineered nano-objects for use in microwave, electronic devices and biomedical sensing application. The Seventh International Symposium on Swift Heavy Ions in Matter, Jun 2008, Lyon, France. hal-00266316

\section{HAL Id: hal-00266316 https://hal.science/hal-00266316}

Submitted on 22 Jul 2008

HAL is a multi-disciplinary open access archive for the deposit and dissemination of scientific research documents, whether they are published or not. The documents may come from teaching and research institutions in France or abroad, or from public or private research centers.
L'archive ouverte pluridisciplinaire HAL, est destinée au dépôt et à la diffusion de documents scientifiques de niveau recherche, publiés ou non, émanant des établissements d'enseignement et de recherche français ou étrangers, des laboratoires publics ou privés. 


\title{
Templates for engineered nano-objects
}

\section{for use in microwave, electronic devices and biomedical sensing application}

\author{
E. Ferain ${ }^{*}$, R. Legras \\ Unité de physique et de chimie des hauts polymères (Université catholique de Louvain) \\ Croix du Sud, 1, 1348 Louvain-la-Neuve, Belgium \\ it4ip sa, rue J. Bordet (Z.I. C), 7180 Seneffe, Belgium
}

\begin{abstract}
Nanoporous templates have been developed and fabricated employing technology based on heavy ion bombardment and track etching of polymer films or polymer layers deposited on substrates; an alternative and unique tool based on the adaptation of an atomic force microscopy has been also developed to elaborate nanotemplates with pores of ultra-small dimensions down to a few nanometers. Different types of nano-objects elaborated using these templates have been further characterized regarding their specific properties : spin dependent phenomena in magnetic nano-objects (GMR, TMR, spin filtering, magnetoCoulomb effect, spin transfer torque phenomena), microwave properties of ferromagnetic nanowires arrays, optical properties of confined emitting polymer and bio-medical applications of magnetic (Ni) and $\mathrm{Ni} / \mathrm{Au}$ composite nanowires. Potential application of magnetic nanowire arrays has been also considered in sensor for automotive contact-less positioning system.
\end{abstract}

*corresponding author : etienne.ferain@uclouvain.be 


\section{Introduction}

Underpinned by results from two previous EC funded projects (PTM\&GMR [1] and NANOPTT [2] ), NANOTEMPLATES project [3] aimed at extending the frontiers of science in the area of making nanotemplates in order (i) to synthesize nano-objects presenting advantageous properties discontinuities arising from the nano-regime and (ii) to explore the potential for innovative and high value added applications. This paper reviews main results obtained in the frame of this project with many references to relevant published scientific papers.

\section{Nanotemplating in polymers}

Nanoporous templates have been developed and fabricated using track etching technology on polymer films or polymer layers deposited on varied substrates like wafer, glass or metal [4-8]. These templates are useful for the synthesis of metallic or polymeric nano-objects (Figure 1) and are therefore used for the growth within their pores of arrays of controlled metallic - single $(\mathrm{Co}, \mathrm{Ni}, \mathrm{Au})$, alloys $(\mathrm{CoFe}, \mathrm{NiFe})$, multilayered $(\mathrm{Co} / \mathrm{Cu})$ - nanowires [9-12]. Supported porous templates have been also prepared from (i) thin polycarbonate or polyimide layer deposited on top of patterned ITO coated glass [13] and used therefore as template for the confinement of emitting polymer into polyLED or OLED devices, and from (ii) thick polycarbonate layers deposited on top of copper or nickel coated PET or PEN film for synthesis of bilayered $\mathrm{Ni}$-Au nanowires with applications in biological field.

Track etching process has been also further fundamentally investigated and track characterization has been considered by in-situ FTIR spectroscopy [14] of heavy ion irradiated polycarbonate film and model compounds, and of consecutive emitted gases. Main results show that only radiochemical processes occur with irradiation doses ranging from $250 \mathrm{kGy}$ to $1 \mathrm{MGy}$. Furthermore, carbonate groups are more sensitive at low energy loss while modification of isopropyl and aromatic groups requires medium to high energy losses with a threshold for the creation of phenol and alkyne groups [15]. If production of $\mathrm{CO}, \mathrm{CO}_{2}$ and $\mathrm{CH}_{4}$ is always observed during polycarbonate irradiation, an energy loss threshold is also observed for emission of ethane and unsaturated molecules [16].

\section{AFM-based nanotemplates}

A unique tool based on the adaptation of an atomic force microscopy to elaborate templates with ultrasmall holes (lateral dimension smaller than $5 \mathrm{~nm}$ ) has been developed (Figure 2) and used to design nanocontacts for spintronics studies $[17,18]$. Based on the use of an electrically controlled indentation, this nanostructuration process is simple and better controlled than standard nanoindentation.

This AFM-based nanoindentation has been applied to different types of insulators (PMMA, photoresists, alumina film, ...) and particles have been therefore synthesized into the resulting holes by 
electrodeposition or by sputtering deposition. Indentation parameters have been optimized and $\mathrm{Au}-\mathrm{Au}$ nanocontacts with a resistance up to $1 \mathrm{kOhm}$ corresponding to size smaller than $1 \mathrm{~nm}$ can be obtained.

These AFM-based nanotemplates are versatile and used to make nanojunctions (magnetic tunnel junction, spin filters, ...) or to connect single nano-object; small area of metallic nanocontacts can be also used to study spin injection, ballistic transport, ... This approach is unique and gives access to the physics of spin polarized transport in ultra-small objects $[19,20]$.

\section{Spin phenomena}

Spintronics has become in less than two decades one of the strongest research fields in solid state physics. The basic of this new area is the control of the carrier motion no more through action on its charge as in classical electronics but rather by acting on its spin.

Giant magnetoresistance (GMR) in magnetic nanowires and nanocontacts [9,21-23]

Experimental set-up to probe the GMR effect with current perpendicular to the plane (CPP-GMR) has been developed combining electrochemical deposition into pores and electron beam lithography. Results obtained on magnetic Co layers are explained in terms of CPP-GMR theory and micromagnetics considerations. Scaling-up to mass production of $\mathrm{GMR} \mathrm{Co} / \mathrm{Cu}$ nanowires arrays has been also evaluated for their potentialities to be applied in the fabrication of GMR sensor for automotive contact-less positioning systems.

\section{Tunnel magnetoresistance (TMR) and spin filtering [19]}

Magnetic tunnel junctions are used as bit cells in MRAM applications and investigation of magnetotransport properties of new materials is strongly considered for the development of the next generation of MRAM. Thanks to the AFM tool, extremely small nano-contacts have been elaborated from new families of magnetic compounds and important advances on the spin dependent properties of complex ferromagnetic insulating oxides have been shown.

\section{Magneto-Coulomb effect [20]}

Fundamental study of spin polarized single electron tunneling (Magneto-Coulomb effect) has been addressed through nanotunnel junctions in which magnetic or non-magnetic nanometric clusters are embedded in an insulating layer. Using AFM tool, a ferromagnetic electrical contact has been obtained on a single isolated nanoparticle and typical magneto-Coulomb effect is observed with $2.5 \mathrm{~nm}$ size nonmagnetic nanoclusters made of $\mathrm{Au}, \mathrm{Al}$ or $\mathrm{Cr}$.

\section{Spin transfer torque phenomenon [17,18]}

As an alternative to lithographic processes, a new reliable and flexible method combining template synthesis and nanolithographic process has been developed to fabricate CPP-GMR spin-valve nanowires 
and to therefore explore electrical spin transfer phenomena. Both magnetic excitations and switching phenomena driven by a spin-polarized current have been demonstrated in electrodeposited $\mathrm{NiFe} / \mathrm{Cu} / \mathrm{NiFe}$ and $\mathrm{Co} / \mathrm{Cu} / \mathrm{Co}$ trilayer nanowires.

\section{Microwave phenomena $[18,24-28]$}

Microwave properties of ferromagnetic nanowires embedded in porous polymer templates have been extensively characterized to demonstrate potentialities to make circulators operating at various frequencies without any external magnetic bias (Figure 3).

FMR properties may be controlled through an appropriate choice of materials, template characteristics and electrodeposition conditions. By tuning these parameters, it is possible to adjust the zero-field resonance frequency from close to zero to up to $36 \mathrm{GHz}$.

The objective of a commercial device is not yet reached but main performance limitation factors were clearly identified and pertinent solutions proposed to increase the performances. New designs have been proposed and evaluated through measurement and simulation; the pertinence of a multi-layer design is proven through modeling and measurement. Design recommendations and material specifications have been clearly established in order to obtain competitive insertion loss level.

\section{Photoluminescence [13]}

The use of nanotemplates was seen as a means which could pave the way for a very interesting phenomenon as the three dimensional confinement of the optical field into a micropillar cavity and its corresponding reduced-mode volume leads to a significant increase of the spontaneous emission rate. A method for the deposition of thin film OLED devices into nanoporous templates has been developed and detailed optical measurements have been made in order to identify narrowing or shift of the emission spectra, and for an increase in the external quantum efficiency (Figure 4).

So far light confinement or quantum confinement effects have been observed but one surprising result coming out of this work is the improved longevity of such devices made on supported templates. Because each macroscopic pixel is effectively made up of millions of individual sub-pixels, i.e. each pore is a pixel, decomposition of the reactive metal cathode is prevented from growing after nucleating at one point in the device, and the degradation of the metal cathode is therefore confined to that pore and does not 'spread' across the surface of the whole pixel. This simple way to prevent cathode degradation without the need to use complex lithography may be extremely useful and is now explored.

\section{Bioanalytical and microfluidics $[29,30]$}

Nanoparticles are known to be very useful in biological applications for tagging or marking cells, DNA, proteins or viruses; for example, magnetic and gold nanoparticles are widely used for the separation or purification of DNA, RNA or protein. Over the course of this project the ability to produce nanowires 
with a range of sizes (100 nm diameter and anything from $500 \mathrm{~nm}$ to $5 \mu \mathrm{m}$ in length) and a variety of compositions - pure nickel, pure gold and part nickel, part gold - has been clearly demonstrated. The nickel component of the nanowires renders them susceptible to an external magnetic field by which the position of the wires could be controlled. Techniques to modify the gold section of the nanowires with DNA, while they were attached to the substrate, have been developed in order to make the wires potentially relevant for clinical diagnostic assays. However, the mass of the wires and their magnetic properties can cause aggregation and sedimentation once they are released from the substrate. The attachment of DNA to the wires does not seem to prevent this phenomenon.

Research and development on microfluidic modules for the application of magnetic nanoparticles in a clinically relevant protocol (BCR-ABL test for chronic myeloid leukaemia - CML) was also carried out; the various steps which are performed in this test are also present in many other molecular biology based diagnostic protocols : cell separation, lysis, RNA extraction, cDNA production from the RNA and quantitative real-time PCR for the analysis of the cDNA. During the project, microfluidic devices (Figure 5) have been designed, built and tested for RNA extraction step and for mRNA conversion to cDNA by reverse transcription; results are promising to significantly reduce the overall sample preparation and assay time. In the future, these microfluidic devices could also make use of the nanoparticles and/or nanowires described in this paper.

\section{Acknowledgments}

The authors are very grateful to the partners involved in the NANOTEMPLATES project and more specially to Frédéric Nguyen Van Dau and Jean-Claude Mage (Thales SA, France), to Tom Harvey and Charles Twist (Epigem Ltd ,UK), to Vincent Cros (CNRS Orsay, France), to Daniele Pullini (Centro Ricerche Fiat, Italy), to Andy Monkman (University of Durham, UK), to Calum McNeil (University of Newcastle, UK) and to Luc Piraux and Isabelle Huynen (Université catholique de Louvain, Belgium). This work was supported by the European Community in the frame of the Sixth Framework Programme under contract $\mathrm{n}^{\circ}$ NMP-CT-2004-505955.

\section{References}

[1] Brite-EuRam 3, Nanoporous particle track etched membranes -ptm- and their use as templates for electrodeposited multilayers for giant magnetoresistance applications - gmr, 1996-1999.

[2] FP5 Growth, Conductive nanowires for applications in microwave, magnetic and chemical sensing devices based on polymer track etched templates, 2000-2003.

[3] FP6 NMP, Templates for engineered nano-objects for use in microwave, electronic devices and biomedical sensing applications 2004-2007. 
[4] L. Vila, P. Vincent, L. Dauginet-De Prac, G. Pirio, E. Minoux, L. Gangloff, S. DemoustierChampagne, N. Sarazin, E. Ferain, R. Legras, L. Piraux, P. Legagneux, Nano Letters 4 (2004) 521.

[5] http://www.it4ip.be.

[6] A.Carretero-Genevrier, N. Mestres, T. Puig, A.Hassini, J. Oró, A. Pomar, F. Sandiumenge, X. Obradors, E. Ferain, Advanced Materials (accepted for publication) (2008).

[7] L. Dauginet-De Pra , E. Ferain, R. Legras, S. Demoustier-Champagne, Nuclear Instruments and Methods in Physics Research B 196 (2002) 81.

[8] S.Xavier, S. Matefi-Tempfli, E. Ferain, S. Purcell, S. Enouz-Vedrenne, L. Gangloff, E.Minoux, L.Hudanski, P. Vincent, J.-P. Schnell, D. Pribat, L. Piraux, P. Legagneux, Nanotechnology 19 (2008) 215601.

[9] M. Darques, L. Piraux, A. Encinas, IEE Transactions on Magnetics 41 (2005) 3415.

[10] A. Saib, D. Vanhoenacker, I. Huynen, M. Darques, L. Piraux, Journal of Physics D : Applied Physics 38 (2005) 2759.

[11] M. Darques, A.-S. Bogaert, F. Elhoussine, S. Michotte, J. de la Torre Medina, A. Encinas, L. Piraux, Journal of Physics D : Applied Physics 39 (2006) 5025.

[12] L. Piraux, M. Darques, S. Michotte, Advances in Science and Technology 51 (2006) 7.

[13] C.-J. Chiang, C. Rothe, M. Rosamond, A. Gallant, E. Ferain, R. Legras, D. Wood, A. Monkman, Proceedings of SPIE 6593 (2007) 65930R.

[14] http://cimap.ensicaen.fr/

[15] F. Dehaye, E. Balanzat, E. Ferain, R. Legras, Nuclear Instruments and Methods in Physics Research B 209 (2003) 103.

[16] F. Dehaye, E. Balanzat, E. Ferain, R. Legras, Investigation of oxidative degradation of bisphenol-A polycarbonate under swift heavy ion irradiation, MRS full meeting (Symposium NN: materials for space applications), Boston, 2004.

[17] T. Blon, M. Mátéfi-Tempfli, S. Mátéfi-Tempfli, L. Piraux, S. Fusil, R. Guillemet, K. Bouzehouane, C. Deranlot, V. Cros, Journal of Applied Physics 102 (2007) 130906.

[18] L. Piraux, K. Renard, R. Guillemet, S. Matéfi-Tempfli, M. Matéfi-Tempfli, V. A. Antohe, S.Fusil, K. Bouzehouane, V. Cros, Nano Letters 7 (2007) 2563.

[19] M. Gajek, M. Bibes, A. Barthélémy, K. Bouzehouane, S. Fusil, M. Varela, J. Fontcuberta, A. Fert, Physical Review B (2005) 020406(R). 
[20] A. Bernand-Mantel, P. Seneor, N. Lidgi, M. Muñoz, V. Cros, S. Fusil, K. Bouzehouane, C. Deranlot, A. Vaures, F. Petroff, A. Fert, Applied Physics Letters 89 (2006) 062502.

[21] F. Elhoussine, L. Vila, L. Piraux, G. Faini, Journal of Magnetism and Magnetic Materials 116 (2005).

[22] D. Pullini, G. Innocenti, D. Busquets, A. Ruotolo, Applied Physics Letters 90 (2007) 133106.

[23] D. Pullini, D. Busquets, A. Ruotolo, G. Innocenti, V. Amigo, Journal of Magnetism and Magnetic Materials 316 (2007) e242.

[24] J.-F. Allaeys, B. Marcilhac, J.-C. Mage, Journal of Physics D : Applied Physics 40 (2007) 3714.

[25] J. Spiegel, J. de la Torre, M. Darques, L. Piraux, I. Huynen, IEEE Microwave and Wireless Component Letters 17 (2007) 492.

[26] A. Saib, M. Darques, L. Piraux, D. Vanhoenacker-Janvier, I. Huynen, Proceedings of the European Microwave Association 1 (2005) 67.

[27] A. Saib, M. Darques, L. Piraux, D. Vanhoenacker-Janvier, I. Huynen, IEEE Transactions on Microwave Theory and Techniques 53 (2005) 2759.

[28] M. Darques, J. Spiegel, J. De la Torre Medina, I. Huynen, L. Piraux, Journal of Magnetism and Magnetic Materials (accepted for publication) (2008).

[29] http://www.epigem.co.uk/.

[30] http://www.ncl.ac.uk/cals/. 


\section{Figure captions}

\section{Figure 1}

From left to right : (i) SEM picture of a typical track-etched polymeric membrane; (ii) SEM picture of NiAu nanowires; the lower part of the wires are Ni, the upper part Au; (iii) SEM picture of nano-OLED after dissolution of the polycarbonate template showing the regularly-shaped micropillars left on the ITO surface; (iv) high contrast TEM picture of a backbone shaped $\mathrm{Co} / \mathrm{Cu}$ nanowire of $30 \mathrm{~nm}$ diameter. (photo courtesy of Epigem Ltd and CRFiat)

\section{Figure 2}

AFM observation of a nano-hole fabricated into an insulator layer deposited on a substrate by means of a AFM nanoindentation tool (left); middle and right pictures are respectively a cross section of this nanohole before and after a 30 seconds low power oxygen plasma. Indentation parameters have been optimized to reach $\mathrm{Au}-\mathrm{Au}$ nano-contact with a very high resistance $(1 \mathrm{kOhm})$ corresponding to contact dimension close to $1 \mathrm{~nm}$. (photo courtesy of CNRS Orsay)

\section{Figure 3}

Schematic view of a circulator based on ferromagnetic nanowire array (left), and performances of measured and modeled circulation (middle). Circulator operates at around $26 \mathrm{GHz}$ using Co nanowires; by tailoring the orientation of the Co c-axis with respect to the nanowire axis via the electrodeposition process, operation frequency can be switched to around $32 \mathrm{GHz}$. This frequency can be also fixed to 15 or $19 \mathrm{GHz}$ by using respectively a NiFe alloy or a CoFe alloy. A special flexible test set-up has been designed to test various architectures and design (right). (photo courtesy of Thales)

\section{Figure 4}

Nano-OLED mounted onto a test rig : visible is the glass substrate with the reflective cathodes of the nine individual pixels; one of the pixels is switched on to emit bright green light. (photo courtesy of University of Durham)

\section{Figure 5}

Microfluidic device for the retention of magnetic beads; in this picture the inlet and outlet channels can be seen as well as the magnet (left). Design of a mixer chip consisting in a chamber with several channels fanning out from it (middle). Heated chip for carrying out enzymatic reactions; thin film heater (orange) covers the channel where most of the liquid is held; green and white wire is the thermocouple for temperature monitoring (right). (photo courtesy of Epigem Ltd) 
Figure 1
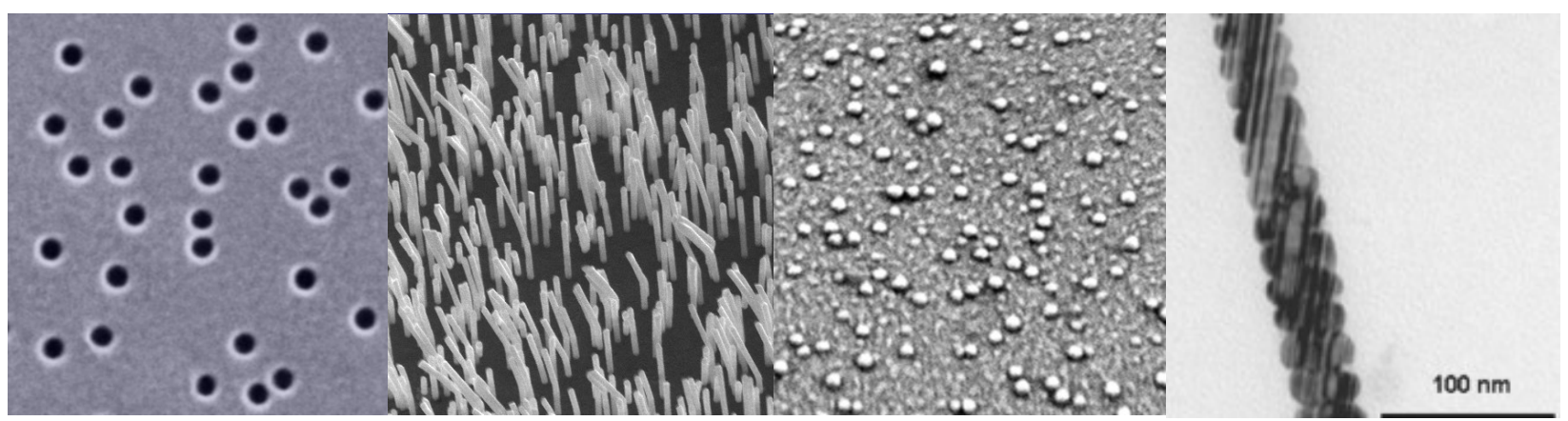

Figure 2
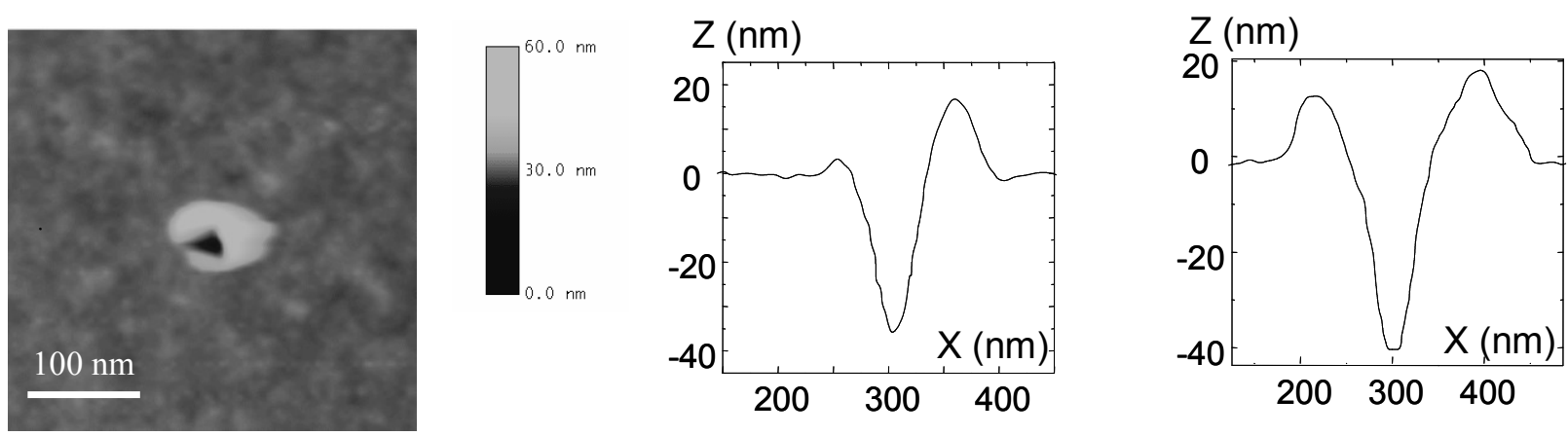
Figure 3
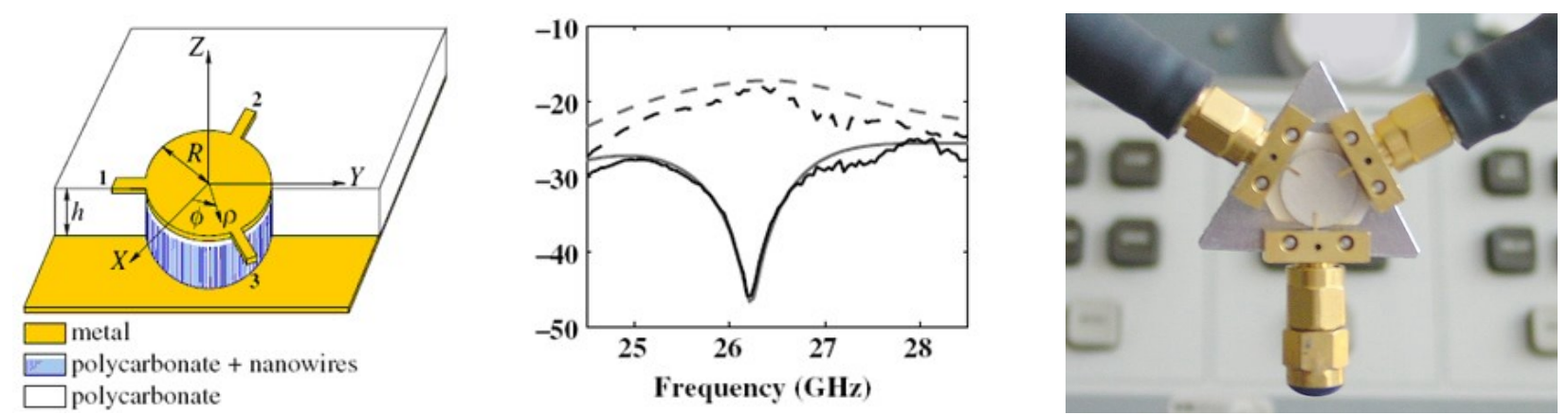

Figure 4

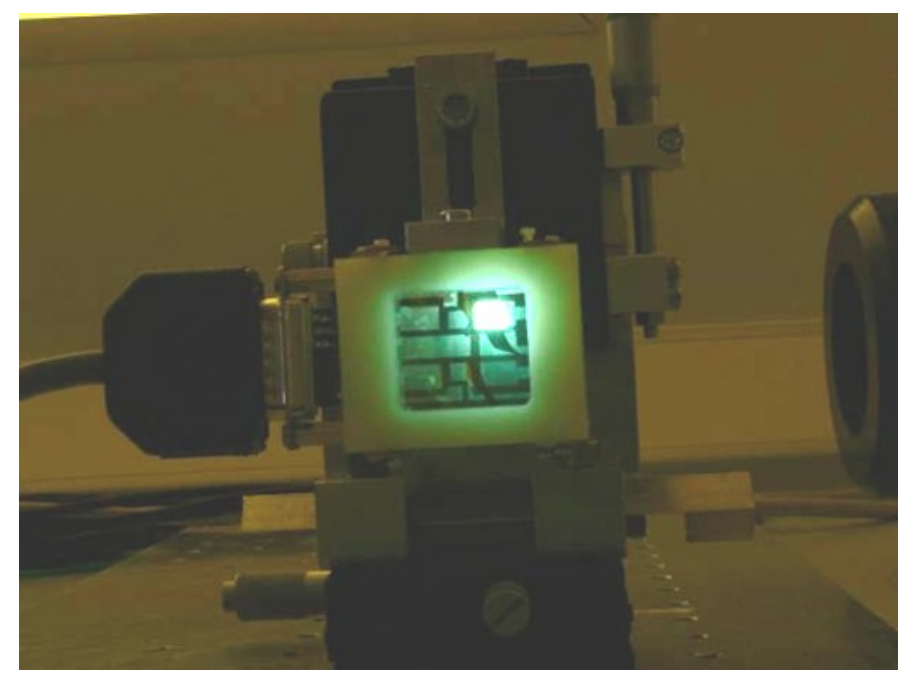


Figure 5
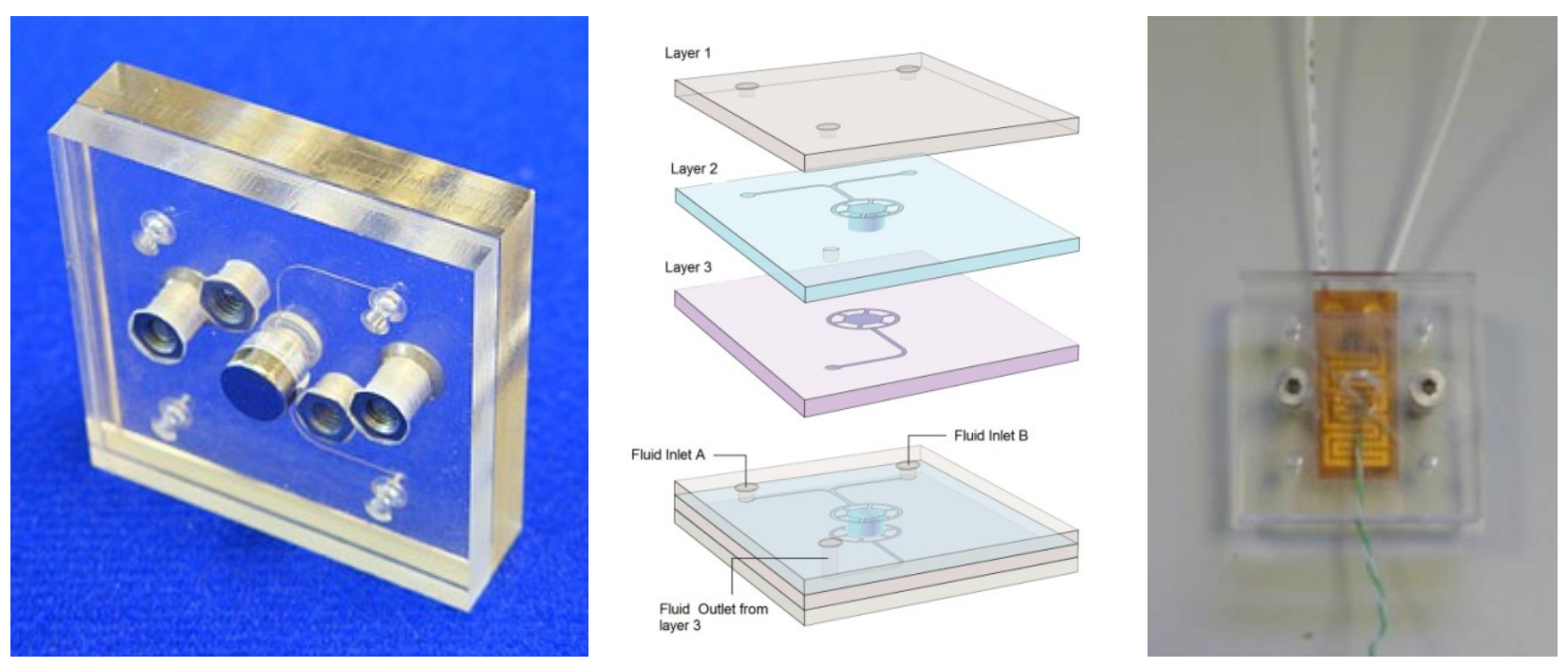